\title{
40. CALCAREOUS ORGANISMS INCERTAE SEDIS FROM THE LOWER CRETACEOUS OF DSDP LEG 27
}

\author{
Hans M. Bolli, Department of Geology, Swiss Federal Institute of Technology, \\ Zurich, and University of Zurich, Switzerland
}

\section{INTRODUCTION}

Small well-preserved spheres with comparatively large, regularly distributed circular pores occur in the eastern Indian Ocean Sites 260, 261, and 263, (Figure 1) where they are restricted to the Lower Cretaceous, Albian/Aptian.

The specimens react very strongly to diluted $\mathrm{HCl}$. Xray examination of two specimens with the Gandolfi camera method, carried out by W. Oberholzer (Zurich) indicates that the tests most likely are made of predominant calcite but may also contain some argonite. Surprisingly, the specimens were found only in virtually non-calcareous sediments where calcareous foraminifera are absent but calcareous nannoplankton is still present, varying from scarce to abundant. Radiolaria and primitive arenaceous foraminifera are also scarce to abundant; dinoflagellates can be abundant and fish remains occur in small quantities.

The faunal and floral composition indicates deposition at a depth where calcareous foraminifera were dissolved but calcareous nannoplankton with its higher resistance was largely unaffected. If this environmental interpretation is correct, it is difficult to understand why these highly calcareous, thin-shelled spheres should have been preserved in such good condition. Contamination from samples with higher $\mathrm{CaCO}_{3}$ content is unlikely because no spheres were seen in many examined samples.

The spheres may be compared to certain Radiolaria, in particular some small spumelline forms, possibly also to representatives of the suborder Phaeodarina, e.g., the Recent but much larger Aulonia hexagonia. The possibility that the spheres could be recrystallized radiolarians $\left(\mathrm{SiO}_{2}\right.$ replaced by $\left.\mathrm{CaCO}_{3}\right)$ was therefore taken into consideration. This, however, seems unlikely for (a) they do not closely compare with known Radiolaria; and (b) all samples containing them also carry Radiolaria with siliceous tests of mostly poor preservation. It is difficult, therefore, to believe that only one Radiolaria taxon of several or many taxa present in a sample should have become recrystallized and at the same time remained much better preserved.

Also considered was the possibility that the specimens could be artifacts of some sort. All samples of the Leg 27 sediments in which the spheres occur were washed for foraminifera and treated with $\mathrm{H}_{2} \mathrm{O}_{2}$. In many of them small tubes of varying shapes were formed by chemical reaction of $\mathrm{H}_{2} \mathrm{O}_{2}$ with small pyritic limonitic nodules present in the sediment. These tubes are non-calcareous and do not resemble the spheres here described. The

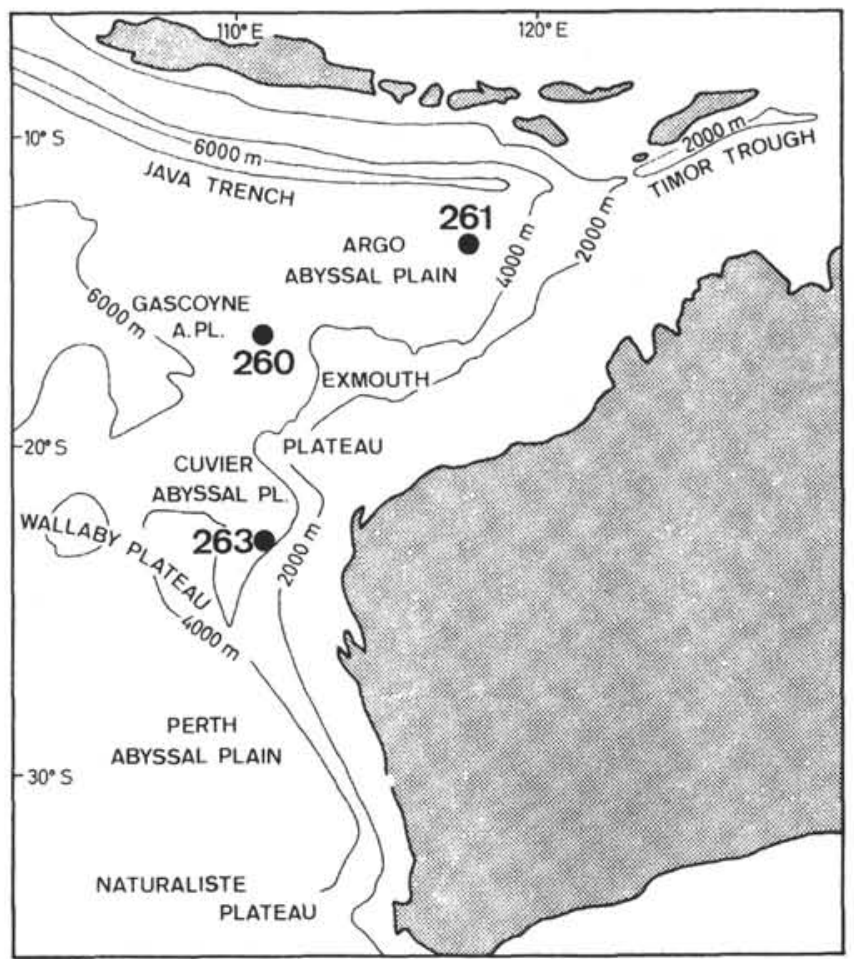

Figure 1. Location of Leg 27 Sites 260, 261 and 263.

rather constant morphology and size of the calcareous spheres can hardly be a product of some chemical reaction.

Though it is not possible to place the spheres with certainty anywhere in the systematic classification at present, they are here reported for documentation. The following description and illustrations by SEM micrographs may aid in their eventual identification.

\section{MORPHOLOGY}

The tests are spherical and occur in two predominant sizes. Smaller specimens measure about $50 \mu$ and larger types about $80 \mu$.

The test wall is penetrated by regularly arranged, more or less circular pores. Most of the pores measure about $5 \mu$ in diameter, with some smaller ones, 3-4 $\mu$. These pores are arranged in a hexagonal pattern so that each pore is surrounded by six others. The wall forming a grid around the pores measures $2.5 \mu-3.5 \mu$ between pores. Under the binocular microscope the surface has a polished, shiny appearance. The grid forms weak, 
rounded, hexagonal ridges around each pore which consequently lie in shallow depressions.

Many of the examined specimens possess a small, faintly conical area without pores. In its center lies a very small opening of less than $1 \mu$ in diameter, which may be surrounded by a rim of variable thickness. A few of the examined specimens of the smaller type possess an extended, rounded, rod-like protuberance. The extension of the specimen shown on Plate 1, Figures 10, 11 measures about $30 \mu$ in length. Its end is rounded with a very minute opening of less than $1 \mu$ in diameter. The outer half of the length of the protuberance measures about $15 \mu$. The base of the high conical inner half is about $25 \mu$ in diameter.

Some broken specimens reveal an inner structure with numerous pillar-like elements which seem to reinforce the sphere. They consist of small, elongate crystals (Plate 1, Figures 9, 12). Other specimens seem to be empty. The nature of these pillars must still be investigated; they may be the result of recrystallization and possibly consist of argonite.

\section{OCCURRENCE}

Specimens of the smaller type (about $50 \mu$ in diameter) occur in all samples listed below, the larger type of about $80 \mu$ in diameter was only seen in the samples of Site 260.

Samples 260-12-1, 86-88 cm $260-13-1,68-70 \mathrm{~cm}$ $260-15-2,32-34 \mathrm{~cm}$ $260-15$, CC $260-16-1,72-74 \mathrm{~cm}$ $260-18-2,84-86 \mathrm{~cm}$

Samples 261-22, CC 261-23, CC

Samples 263-3, CC

263-6, CC

263-7, CC

263-8, CC

263-9, CC

263-12, CC

Specimens are scarce to very scarce in all samples except for Sample 260-18-2, 84-86 cm, where they are fairly frequent. For accompanying faunas and floras and the ages indicated by them, see Figures 2, 3, 5 in the Synthesis of the Leg 27 Biostratigraphy and Paleontology (this volume).

The specimens figured on Plate 1 are deposited at the Museum of Natural History, Basel, under the numbers C 30141-30147. 


\section{PLATE 1 \\ SEM micrographs \\ of calcareous organisms incertae sedis}

Figures 1, 2, 7, Small type, about $50 \mu$ in diameter.

$8,10,11$ 1. Sample 260-15, CC; $\times 500$; C 30141.

2. Sample $260-18-2,84-86 \mathrm{~cm} ; \times 500$; with low conical area without pores but with very small opening (upper center); C 30142.

7. Detail of lower left of Figure 2 ; $\times 2500$; displays hexagonal patterns of pore arrangement and weak hexagonal ridges surrounding each pore.

8. Detail of central area of Figure $1 ; \times 2000$; displays hexagonal ridges surrounding each pore. 10. Sample $260-15-2,32-34 \mathrm{~cm} ; \times 500$; type with large poreless protuberance; C 30143 .

11. $\times 1000$; detail of Figure 10; very small opening at end of protuberance visible.

Figures 3-6, Large type, about $80 \mu$ in diameter.

9,12

3. Sample 260-15, CC; $\times 450 ;$ C 30144.

4. Sample $260-15, C C ; \times 500$; with low conical area without pores but with small opening (upper center); C 30145.

5. Sample $260-18-2,84-86 \mathrm{~cm} ; \times 500$; with low conical area without pores but with small opening (upper center); C 30146.

6. Detail of upper center of Figure $5 ; \times 1000$; with low conical area without pores and small opening surrounded by distinct rim.

9. Sample $260-15, C C ; \times 500$; inside view of broken specimen with pillar-like elements, possibly a result of recrystallization; C 30147.

12. Detail of Figure $9 ; \times 1000$. 
PLATE 1
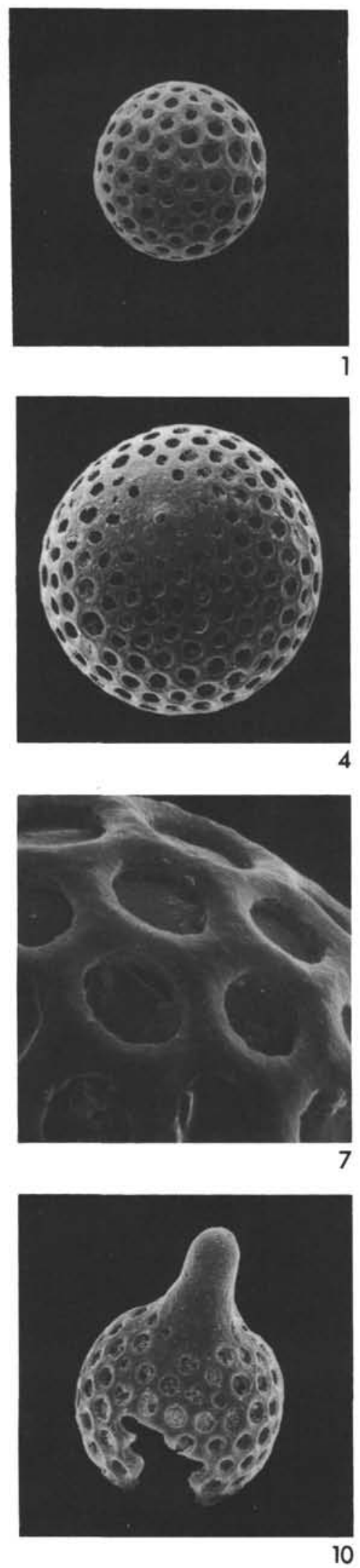
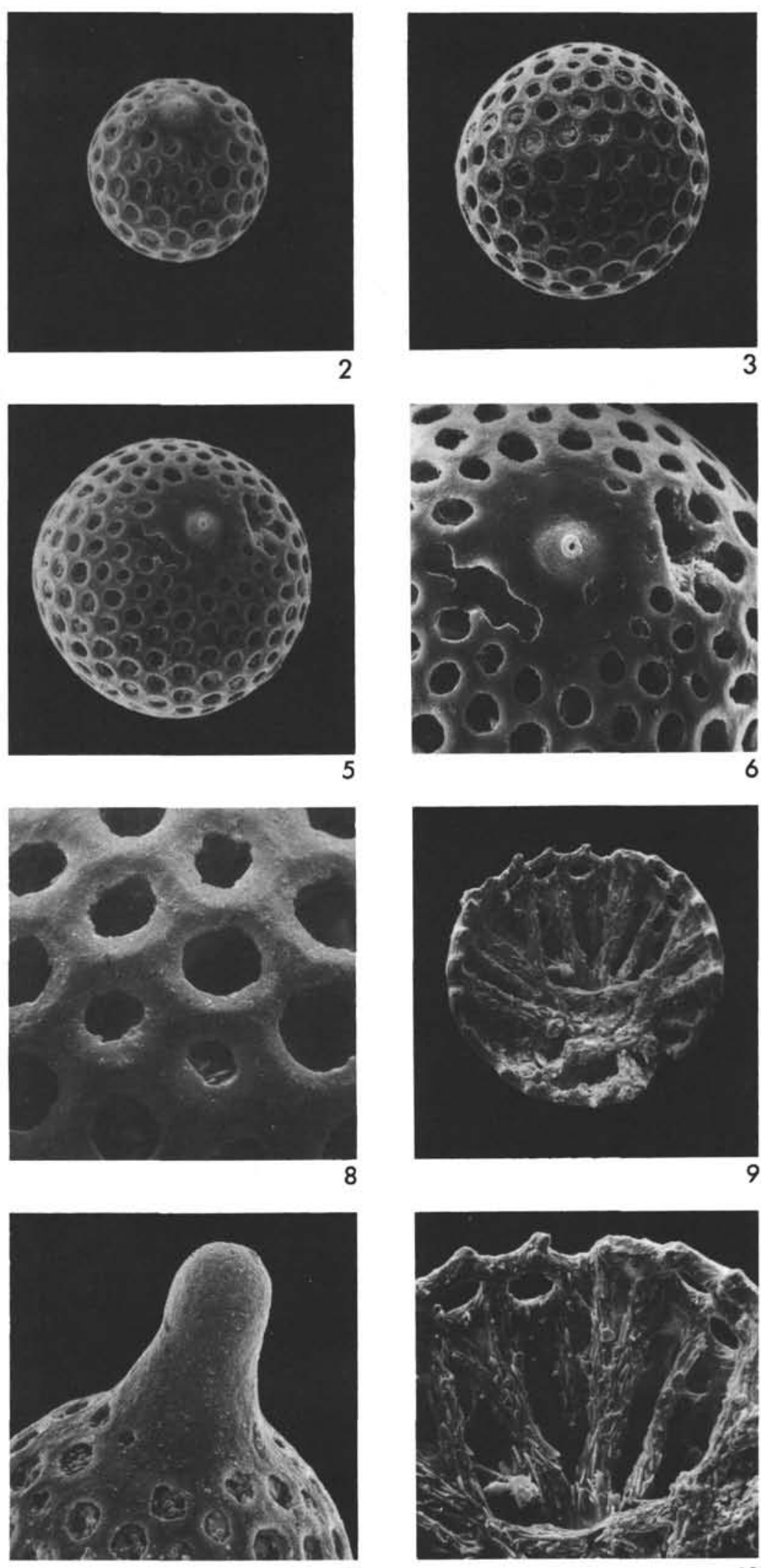

11

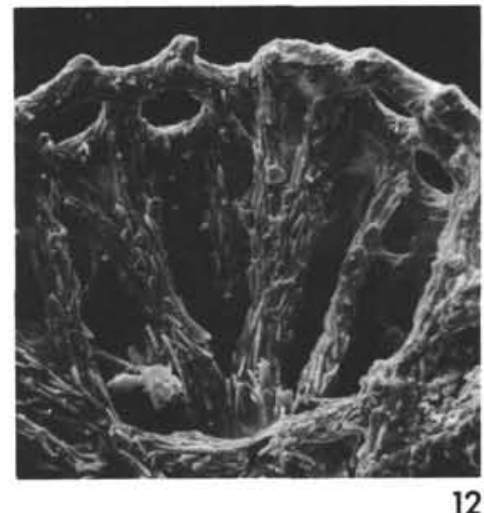

